1 Department of operative dentistry, School of dentistry, Alborz University of Medical Sciences, Karaj, Iran

${ }^{2}$ Faculty of dentistry, Hamedan University of Medical Sciences, Hamedan, Iran

${ }^{3}$ Faculty of dentistry, Tehran University of Medical Sciences, Tehran, Iran

4 Department of Medical Surgical Nursing, School of Nursing and Midwifery, Shahid Beheshti University of Medical Sciences, Tehran, Iran

Corresponding author: Bahareh Asgartooran Faculty of dentistry, Hamedan University of Medical Sciences, Hamedan, Iran

Email:asgartooranbahareh@gmail.com Phone: +982182401615

Fax: +982182401675

Received: October 07, 2018

Accepted: March 22, 2019

\section{Effect of Modified 45s5 Bioglass on Physical and Chemical Properties of Bleached Enamel}

\author{
Mahshid Saffarpour ${ }^{1}$, Bahareh Asgartooran ${ }^{2,}$, \\ Mohammad reza Tahriri ${ }^{3}$, Maryam Mohammadi \\ Savadroudbari ${ }^{1}$, Mehdi Khabazkhoob ${ }^{4}$
}

Aim: The purpose of this study was to evaluate of the effects of modified $45 S 5$ bioglass (BG) before, after, and during the bleaching procedure with $35 \%$ hydrogen peroxide (HP) on the tooth colour change and physicochemical and morphological properties of human enamel. Methods: Forty-two human premolar enamel samples were prepared and randomly divided into six groups as G1: control (deionized distilled water for $20 \mathrm{~min}$ ), G2: BG (Bioglass suspension for $20 \mathrm{~min}$ ), G3: HP (hydrogen peroxide $35 \%$ for $20 \mathrm{~min}$ ), G4: BG before HP (Bioglass suspension for $20 \mathrm{~min}$ followed by hydrogen peroxide 35\%), G5: BG after HP (hydrogen peroxide 35\% followed by Bioglass suspension for $20 \mathrm{~min}$ ), and G6: BG during HP (Bioglass in hydrogen peroxide 35\% suspension for $20 \mathrm{~min}$ ). The treatment procedure was performed on the whole enamel surface. Colorimetry was done before and after the treatment procedure. Two specimens from each group were selected for morphological analysis with scanning electron microscope (SEM). Microhardness analysis was performed after the treatment procedure and chemical analysis of BG dissolution was done for BG+DDW and BG+HP suspensions. Results: No statistically significant difference in colour was observed among different groups $(P=0.073133)$ and the yellowness index decreased in all of the four HP groups. The greatest reduction in microhardness occurred in groups HP and BG before HP $(P<0.001)$ while the BG group showed increased microhardness measurements $(P<0.001)$. Statically significant differences in microhardness were found among the groups. Elemental analysis showed significantly increased levels of $\mathrm{Ca}$ and $\mathrm{P}$ in $\mathrm{BG}$ after HP and BG before HP groups when compared to the HP group. Ionic release of BG was significantly greater in HP when compared to DDW. Conclusion: Using BG before HP had a greater protective effect since it increased microhardness more effectively, decreased mineral loss, and retained the integrity of the enamel surface. The HP group had the lowest microhardness and BG during HP showed less protective effects compared to BG before HP.

Keywords: Biocompatible materials. Hardness. Thooth bleaching. Dental enamel. 


\section{Introduction}

Tooth discoloration may compromise smile aesthetics and increase the demand for aesthetic dental treatments. Among various treatment options, tooth bleaching has rapidly gained popularity as an easy and conservative treatment modality for discoloured teeth ${ }^{1-3}$. Hydrogen peroxide $(\mathrm{HP})$ is commonly used as an active agent for dental bleaching. It acts as a strong oxidizing agent through the formation of free radicals, reactive oxygen species, and HP anions. These reactive molecules attack the long-chained, dark-coloured chromophore molecules and split them into smaller, less coloured, and more diffusible molecules ${ }^{2,4}$. Although the efficacy of tooth bleaching has been confirmed, the safety of this technique is under question. A primary concern is that the structure of the sound enamel may be weakened by bleaching agents ${ }^{1}$. They have a direct effect on the protein content of the tooth and can also change the mineral phase of the enamel, leading to chemical and mechanical changes in enamel surface ${ }^{5}$. Some studies have reported no or small morphological alterations in the bleached enamel, but others have shown that porosity, depression, erosion, and roughness increase after applying bleaching agents to the enamel. Enamel hardness has also been evaluated with contradicting results ranging from no effect to a significant decrease in hardness and fracture resistance of the enamel ${ }^{2,4}$. An in vitro study showed that peroxide compositions removed mineral structures from the enamel and these alternations could be observed beneath the surface ${ }^{6}$. Additionally, demineralization of the bleached enamel is not limited to the enamel surface and extends to the enamel subsurface, as well. Therefore, it is necessary to minimize the potential damage to the enamel caused by HP exposure ${ }^{1,7}$. Bioglass (BG) has been shown to inhibit and reverse the progression of initial enamel caries. Studies have indicated the potential of BG in preventing and restoring the enamel defects induced by bleaching agents. BG forms a protective layer on the enamel surface to inhibit demineralization when used prior to bleaching with HP or enhances remineralization when used after HP bleaching. In contact with saliva or any other fluid, BG can release ions and increase the $\mathrm{pH}$; it may also buffer the acidity of $\mathrm{HP}$ and reduce demineralization when mixed with $\mathrm{HP}^{1,8,9}$.

The $45 \mathrm{~S} 5 \mathrm{BG}$ (developed by Hench et al) contains 45\% SiO2, 24.5\% Na2O, 24.5\% CaO, and $6 \%$ P20 5 by weight. It is highly biocompatible, osteoconductive, and osteoinductive. In aqueous media, this material can form hydroxyl carbonate apatite ( $\mathrm{HCA})$ that is similar to biologic inorganic material and is widely used in tissue engineering 1,5,10.

Recently, studies have shown that when strontium is substituted for $\mathrm{Ca}$, the glass network expands to a larger ionic radius of Sr when compared to $\mathrm{Ca}$. By substituting $\mathrm{Sr}$ for $\mathrm{Ca}$, glass degradation and apatite formation can be increased. Apatite formation can be significantly enhanced by fully substituting $\mathrm{Ca}$ for $\mathrm{Sr}^{11,12}$. Recently, BG has also been used in dentistry as a remineralizing agent incorporated in toothpastes, particularly for treating dentin hypersensitivity, since it precipitates HCA onto the tooth surface and seals the dentinal tubules ${ }^{13,14}$. Incorporation of $\mathrm{Sr}$ into BG is useful since $\mathrm{Sr}$ has been shown to prevent dental caries and improve enamel remineralization. Sr salts (such as strontium acetate or strontium chloride) are already used in remineralizing toothpastes ${ }^{11}$. 
The purpose of this study was to evaluate the effects of applying modified 45S5 BG with $\mathrm{Sr}$ before, after, and during tooth bleaching with 35\% HP on the colour and physicochemical and morphological properties of the human enamel.

The null hypothesis of this study was that applying modified BG with Sr before, after, and during bleaching with $35 \%$ hydrogen peroxide has no effects on the colour, microhardness, and morphology of the human enamel.

\section{Material and Methods}

Specifications of BG: The BG powder was characterized by scanning electron microscopy coupled with energy-dispersive X-ray (SEM-EDX) to determine the morphology and ingredients of the Bioglass. X-ray diffraction (XRD) was used for crystalline phase assessment, and Fourier-transform infrared spectroscopy (FTIR) was applied for spectral absorption using Potassium Bromide (KBr).

The modified BG powder was manufactured by a dental material specialist. BG in the system $\mathrm{SiO}_{2}-\mathrm{CaO}-\mathrm{P}_{2} \mathrm{O}_{5}-\mathrm{SrO}$ were prepared using a melt-quench route. $5 \%$ of $\mathrm{Ca}$ were replaced by $\mathrm{Sr}$ on molar base in order to maintain the structure of the silicate glass matrix. Mixture of BG were melted in a platinum-rhodium crucible for $1.5 \mathrm{~h}$ at 1400 in an electric furnace. After melting the glass were rapidly quenched into water. After drying, the glass was ground using vibratory mill for 7 min and sieved to a particle size 10-90 $\mu \mathrm{m}$. The amorphous structure of the glasses was confirmed by powder $X$-ray diffraction.

Preparation of enamel samples: In this study, 42 samples prepared from human premolars were used and stored at $4^{\circ} \mathrm{C}$ in $0.1 \%$ thymol solution until use. Their crowns were cut from the roots using a high-speed handpiece under air-water cooling. Then, each crown was divided into buccal and lingual halves and the specimens were embedded in orthodontic resin. The labial surfaces were serially ground using SiC abrasive papers (600, 1000 and 2000 grit) and then polished with felt cloth impregnated with diamond paste $(1-5 \mathrm{~m} \mu)$. Then, the specimens surfaces were cleaned with DDW for 5 minutes and examined with a stereomicroscope to exclude samples with cracks. Then, the specimens were stored in DDW until use.

Treatment procedure: The specimens were randomly divided to six treatment groups. In G1 (control), $1 \mathrm{gr}$ distilled deionized water (DDW) was applied to the whole enamel surface for 20 minutes. In G2 (BG), 1 gr Bioglass powder in 10 cc DDW was applied for 20 minutes. In G3, HP (35\% hydrogen peroxide) was applied for 20 minutes. In G4, (BG before HP), Bioglass suspension was applied for 20 min followed by applying hydrogen peroxide for 20 minutes. In G5 (BG after HP), hydrogen peroxide was applied for 20 min followed by applying Bioglass for 20 minutes. In G6 (BG + HP), Bioglass and hydrogen peroxide were simultaneously applied for 20 minutes (1 gr Bioglass powder in $10 \mathrm{cc}$ hydrogen peroxide). The materials were applied on the whole enamel surface in all groups. During the treatment procedure, the specimens were incubated at $37^{\circ} \mathrm{C}$ with $95 \% \pm 5 \%$ relative humidity. Between the treatment procedure, the specimens were rinsed with air-water spray for 15 seconds and covered with cotton tissues containing DDW to avoid dehydration. The treatment procedure was repeated in triplicates so that the total application time of 35\% HP (Whiteness Hp Maxx, FGM, Joinville - SC Brazil) was 60 minutes. 
Color change: The colorimetric scales were measured using a spectro-photo-radiometer (CS2000, Konica Minolta, Japan) in a dark room using wavelengths of 380-780 nm at $1 \mathrm{~nm}$ intervals with $45^{\circ} / 0^{\circ}$ geometry. The $2.4 \mathrm{~mm}$ circular center of each specimen was analyzed using the Commission internationale de l'éclairage system (CIE L*a*b*) under standard illumination (D65) at $70 \mathrm{~cm}$ working distance, $0.2^{\circ}$ observer angle, and $45^{\circ} / 0^{\circ}$ geometry. Each specimen was analyzed in triplicate.

Surface microhardness testing: After the treatment procedures, the $\mathrm{SMH}$ of the sound enamel was measured with a Vickers diamond indenter (Laizhou Huayi Testing Instrument Co., Ltd., Shandong, China) using 300g load and 15 seconds dwell time. Three indentations, spaced $50 \mathrm{~mm}$ perpendicularly from each other, were made at the center of the samples.

SEM-EDX analysis: After SMH, two samples from each group were selected for SEM preparation. These samples were cleaned with DDW for five minutes, air-dried, and gold sputter-coated. Morphological changes of the enamel surface were inspected with a scanning electron microscope (XL30 ESEM, Philips, Amsterdam, Netherlands).

Additionally, two samples were prepared and treated with the similar technique as in either BG or BG and HP groups. Then, without cleaning, the samples were directly air-dried and examined with an SEM to observe BG precipitation.

Chemical assessment of BG dissolution: Chemical analysis was performed for BG+DDW (1 gr Bioglass powder in $10 \mathrm{cc}$ DDW) and BG+HP suspensions (1 gr Bioglass powder in $10 \mathrm{cc}$ hydrogen peroxide). The filtrates of the suspensions were analyzed for ionic release by serial passage through a $0.22 \mu \mathrm{m}$ Whatman filter. The free $\mathrm{Ca}, \mathrm{Na}$, $\mathrm{Si}, \mathrm{P}$ and $\mathrm{Sr}$ ions were measured using inductively coupled plasma atomic emission spectroscopy (ICP-AES, Zarian, Australia) in triplicate for each element.

Statistical analysis: Statistical analysis was done using SPSS version 16 (SPSS for Windows, Version 16.0. Chicago, SPSS Inc) Data normality and variance homogeneity were tested by the Kolmogorov-Smirnov test. The SMH and color change $(\triangle \mathrm{E})$ were analyzed between groups using one-way ANOVA. The Kruskal-Wallis test was applied to compare the results of EDX analysis between different groups. The significance level was set at 0.05 for all analyses.

\section{Results}

Specifications of BG: Figure 1a presents the SEM image of BG. The morphology of the powder was specified by fine and irregular particles. The XRD pattern showed a peak centered at $2 \theta \approx 32^{\circ}$ with a wide shape and low intensity, demonstrating the amorphous state of BG. An increase was observed in crystallization the percentage of Sr increased (Figure 1b). The FTIR spectrum is shown in Figure 1c. The bands at 1016, $36 \mathrm{~cm}^{-1}$ were related to Si-O-Si.

Color change: The positive yellowness index changed in all groups, indicating that all four bleaching treatments effectively decreased the yellowness index. The yellowness index was calculated according to ASTM E313 standard and reduction of the yellowness index was considered as whiteness yellowness index calculated as $\Delta \mathrm{YI}=\mathrm{YI} 1$ (before treatment) - YI2 (after treatment). Moreover, the yellowness index was signifi- 

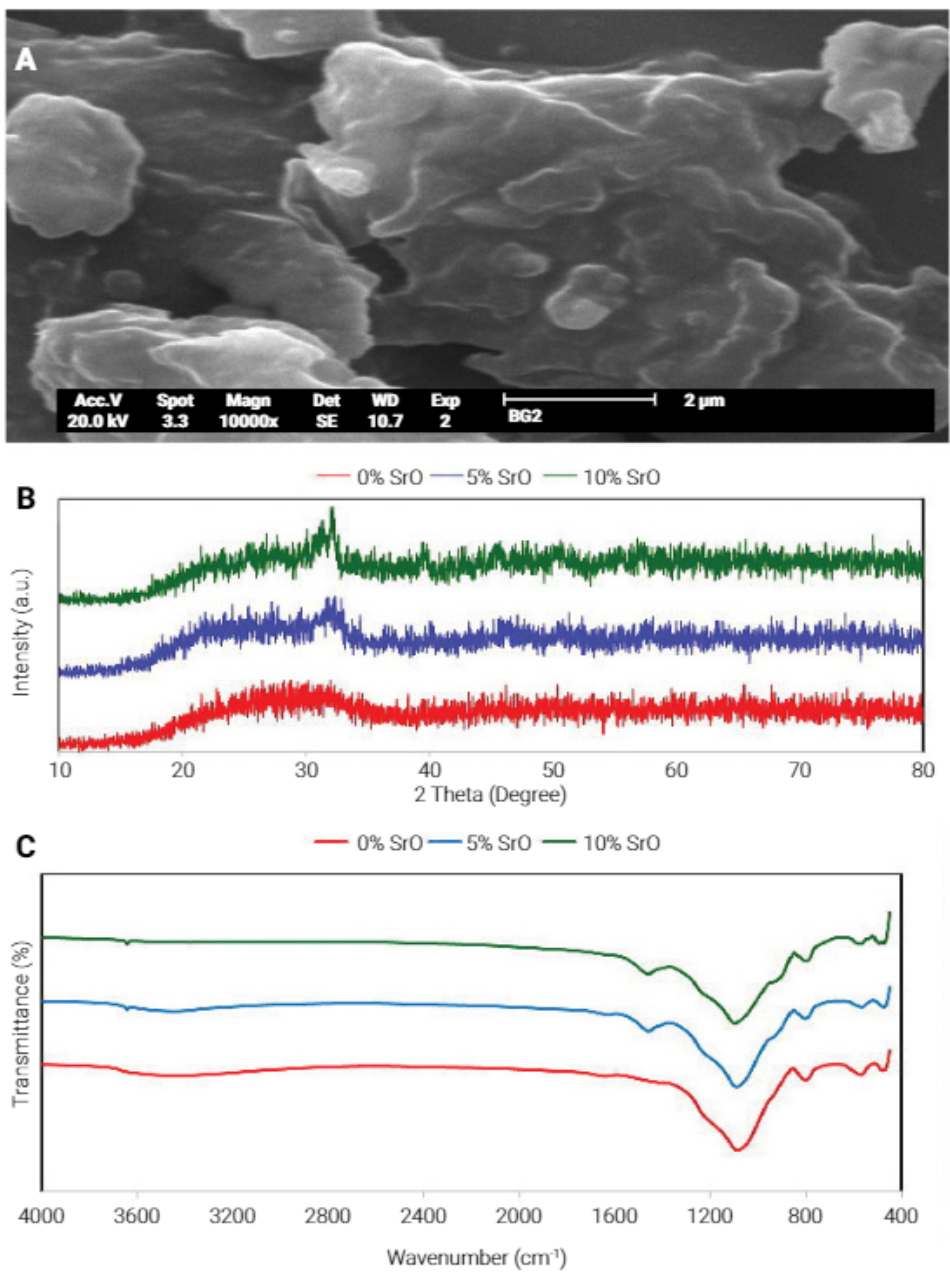

BG: Bioglass; FTIR: Fourier-transform infrared spectroscopy; SEM: Scanning electron microscopy; XRD: X-ray diffraction.

Figure 1. Characterization of BG. (a) SEM image; (b) XRD pattern; (c) FTIR spectrum

cantly higher in groups HP and BG after HP compared to the groups BG and BG+HP (Figure 2). No statistically significant differences were found in $\triangle E$ between $B G$ and $\mathrm{BG}+\mathrm{HP}(\mathrm{P}=0.073133)$.

Surface microhardness: Surface microhardness values are shown in Table 1 and Figure 3. The SMH of BG before HP, BG, and control groups was significantly higher than that of BG after HP, HP, and HP+BG groups. The SMH was lower in the HP group. One-way ANOVA showed statistically significant differences in the surface microhardness between different groups $(P<0.010)$. The results of post hoc ANOVA test are shown in Figure 3.

SEM-EDX analysis: The SEM images of the enamel surface morphology are presented in Figure 4. The enamel surfaces remained unchanged in the control, BG, and BG before HP groups (Figures $4 a, 4 b$ and $4 d$ ). The most evident morphological changes in the enamel surface (demineralization) were seen in the HP group 


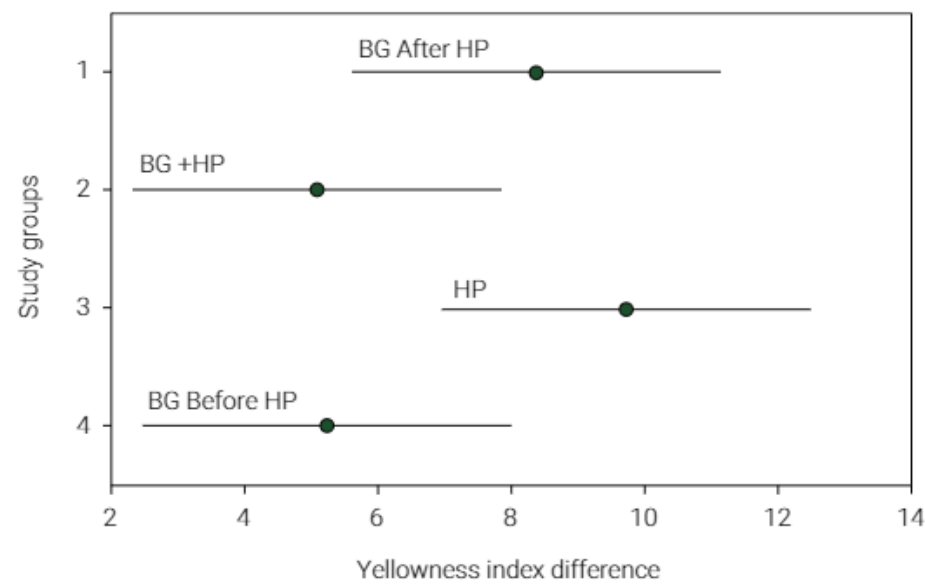

Figure 2. Results of post hoc ANOVA for the difference in yellowness index (groups HP and BG after HP in the right side)

Table 1. The mean and standard deviation of surface microhardness

\begin{tabular}{cccc}
\hline & & \multicolumn{2}{c}{ Surface microhardness(Unit, Kg/mm2) } \\
\cline { 2 - 4 } & & Mean & Standard deviation \\
\cline { 2 - 4 } & Control & 308.92 & 26.26 \\
\cline { 2 - 4 } Group & HP & 267.03 & 12.01 \\
\cline { 2 - 4 } & BG before HP & 316.80 & 43.93 \\
\cline { 2 - 4 } & BG after HP & 300.51 & 15.70 \\
\hline & HP and BG & 286.90 & 28.72 \\
\hline
\end{tabular}

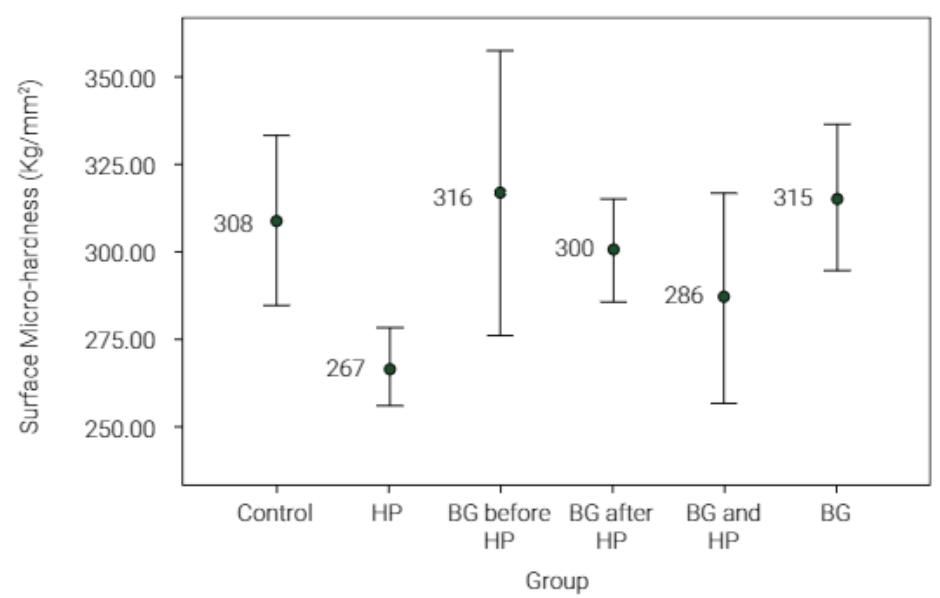

Figure 3. The mean and $95 \% \mathrm{Cl}$ for surface microhardness of study groups

(Figure 4c), ranging from a mild increase in the surface porosity and slight etching along with increased surface irregularities to severe destruction of the enamel surface causing significant depressions and irregularities (Figure 4c). Mild morphological changes were detected in groups BG after HP and BG+HP (Figure 4e and 4f). The 

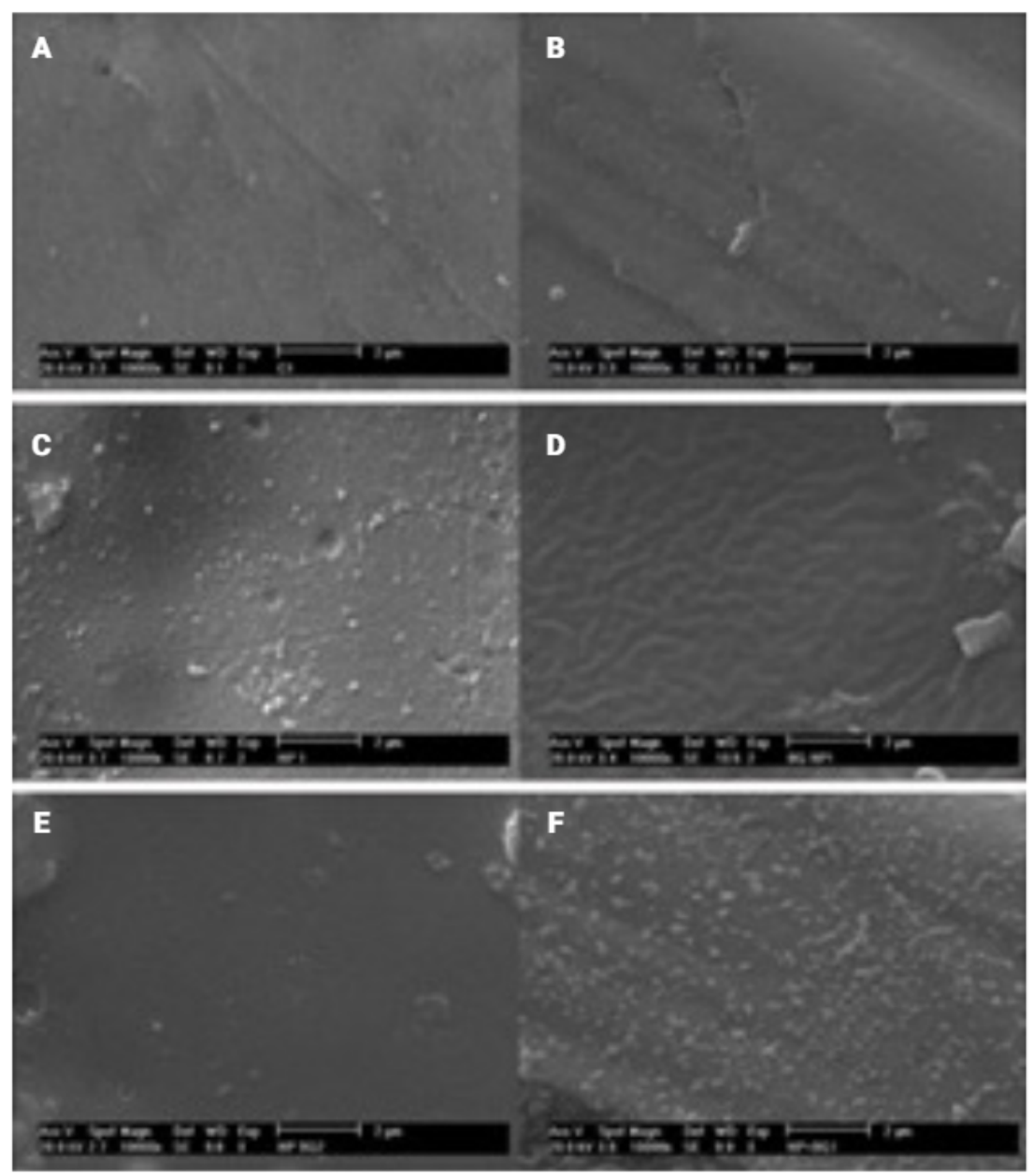

BG: bioglass; DDW: Deionized distilled water; HP: Hydrogen peroxide; SEM: Scanning electron microscopy Figure 4. SEM images $(\times 10,000)$ of enamel surfaces subjected to different treatments. (a) DDW group; (b) BG group; (c) HP group; (d) BG before HP group;(e) BG after HP group; (f) BG during HP group.

group that was first treated with HP and then with BG showed a completely different surface appearance (Figure 4e).

The SEM images showed precipitation of BG particles diffused over the enamel surface covering the porosities of the bleached enamel rather thoroughly (Figure 5).

Figure 5 a shows the BG deposits on the enamel surfaces treated with BG+DDW (Figure $5 a$ and $5 b$ ) and BG+HP (Figure $5 c$ and $5 d$ ). The treated enamel surfaces were sealed with $B G$ particles, and BG particles from the BG+HP suspension were more diffused than those from the BG+DDW suspension.

According to results of the EDX analysis, the lowest and highest mean values of $\mathrm{Ca}$ and $P$ were seen in the HP and BG group without any significant difference $(P<0.069)$. The results of the EDX elemental analysis confirmed that remineralization occurred in the experimental groups, since there were increased levels of $\mathrm{Ca}$ and $\mathrm{P}$ when compared to the HP group. (Table 2). 
Chemical analysis of BG dissolution: The results of the ionic release profile of BG are shown in Table 3 . The ionic release of BG was significantly greater in HP when compared to the DDW group.
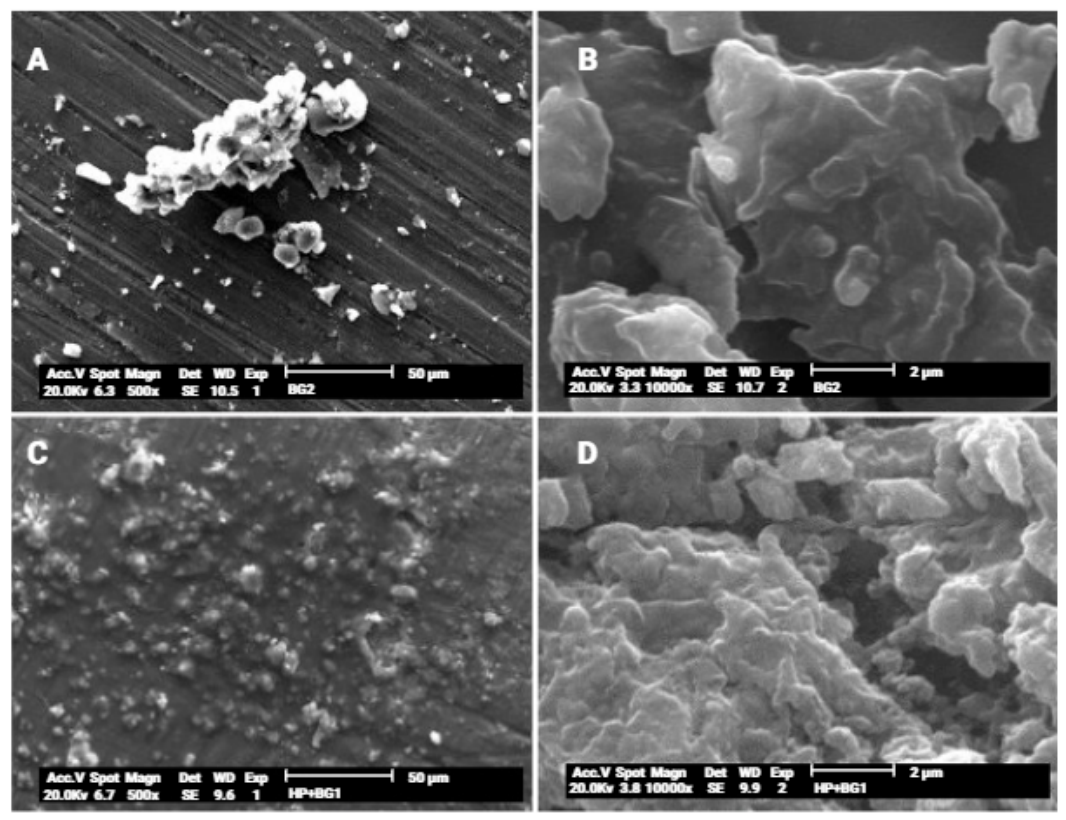

BG: Bioglass; DDW: Deionized distilled water; HP: Hydrogen peroxide; SEM: Scanning electron microscopy. Figure 5. SEM images of BG remaining on the enamel surface. $(\mathrm{a}, \mathrm{b})$ : Treated with BG+DDW at $\times 500$ (left) and $\times 10,000$ (right) magnifications. BG: Bioglass; DDW: Deionized distilled; (c, d): Treated with BG+HP suspensions at $\times 500$ (left) and $\times 10,000$ (right) magnifications.

Table 2. Ratio of $\mathrm{Ca}$ to $\mathrm{P}$ in different groups

\begin{tabular}{lcc}
\hline Group & Ca/P mean & P value \\
\hline DDW & 2.05 & \\
\hline BG & 2.68 & \\
\hline HP & 1.35 & $0.069^{\star}$ \\
\hline BG before HP & 1.82 & \\
\hline BG after HP & 1.44 & \\
\hline
\end{tabular}

*The $\mathrm{P}$ values were calculated by the Kruskal-Wallis test.

Table 3. Ionic release profile of $\mathrm{BG}+\mathrm{DDW}$ and $\mathrm{BG}+\mathrm{HP}$ groups

\begin{tabular}{lccccc}
\hline Group & $\begin{array}{c}\text { Ca ppm } \\
(396.847 \mathrm{~nm})\end{array}$ & $\begin{array}{c}\text { Na ppm } \\
(589.592 \mathrm{~nm})\end{array}$ & $\begin{array}{c}\text { P ppm } \\
(189.925 \mathrm{~nm})\end{array}$ & $\begin{array}{c}\text { Si ppm } \\
(251.611 \mathrm{~nm})\end{array}$ & $\begin{array}{c}\text { Sr ppm } \\
(216.596 \mathrm{~nm})\end{array}$ \\
\hline BG+DDW & 64.2 & 0.13 & 9.2 & 120.9 & 77.2 \\
\hline BG+HP & 1805 & 20.01 & 324.3 & 44.1 & 518.8 \\
\hline
\end{tabular}




\section{Discussion}

Although bleaching is a common dental procedure, controversies exist regarding its side effects. There are different reports about the capability of bleaching agents to cause morphological alternations in the enamel. These changes are related to the length of the bleaching process and the concentration of the bleaching agent ${ }^{3}$.

There are different at-home and in-office bleaching techniques. The at-home bleaching technique requires the application of low concentrations of HP or carbamide-peroxide (CP) for weeks. During this time, demineralization and remineralization will occur ${ }^{1,13}$. In-office bleaching is based on the use of high concentrations of HP. In this study, we used a high concentration of HP to prevent the effect of demineralization/remineralization cycles on the effect of $\mathrm{BG}^{1}$.

In this study, we used modified $45 \mathrm{~S} 5$ Bioglass (containing Sr). Several studies have shown that substituting $\mathrm{Sr}$ for $\mathrm{Ca}$ increases the glass dissolution and ionic release, and effect that depends on the expansion of the glass network due to the larger ionic radius of $\mathrm{Sr}$ ions compared to $\mathrm{Ca}$. Furthermore, $\mathrm{Sr}$ substitution increases $\mathrm{Sr}$ release, and apatite formation is significantly improved in the completely Sr substituted glass, which enhances osteoblast attachment and increases the control over osteoblast/osteoclast activity, as shown previously ${ }^{11,12}$.

Nowadays, BG is practiced in dentistry as a remineralizing material added to toothpastes, particularly for eliminating dentin sensitivity by depositing HCA onto the tooth surface and covering dentinal tubules ${ }^{13,14}$. Incorporation of Sr in BG is useful, as $\mathrm{Sr}$ has been shown to prevent dental caries $^{15}$ and improve enamel remineralization. Sr salts (such as strontium acetate or strontium chloride) are already used in remineralizing toothpastes ${ }^{16}$.

Moreover, bioactive glasses have the potential to improve the beneficial effects of apatite formation with a more controlled release of $\mathrm{Sr}$ ions for eliminating dentin hypersensitivity and preventing caries ${ }^{4}$.

One of the objectives of this study was to evaluate the effect of BG on the bleaching efficiency of HP on the tooth enamel. Active oxygen radicals produced by HP breakdown destroy the double bonds and oxidize the chain of the chromophore, resulting in tooth whitening ${ }^{1,17}$. The whitening procedure can be evaluated using shade guides or a spectrophotometer; the latter is more objective and reproducible for quantitative evaluation of tooth color change ${ }^{18}$.

In the current study, all bleaching groups showed a typical color change of increased $L *$ and decreased $b *$ values, which was consistent with the results of effective bleaching treatments reported in previous studies $1,13,19$.

In this study, all bleaching groups showed a reduction in the yellowness index although this reduction was more obvious in $\mathrm{HP}$ and $\mathrm{BG}$ after $\mathrm{HP}$ groups compared to $\mathrm{BG}$ before HP and BG during HP groups. However, the hypothesis that BG precipitation can disrupt the penetration of peroxide and decrease its color change was rejected in this study because $\Delta \mathrm{E}$ values were equal in all bleaching groups ${ }^{1}$. 
Increased enamel mineral loss and formation of pits, depressions, and porosities in the enamel surface have been reported after HP bleaching ${ }^{13,17,20}$. Microhardness is a simple and confident method for evaluation of the inorganic content alternation of the hard tissue. It is also an indirect measure of the inorganic alternation of the enamel surface after bleaching ${ }^{6}$. The oxide reduction reaction of the bleaching agent can lead to dissolution of both organic and inorganic dental matrices, ultimately leaving only carbon dioxide and water ${ }^{21}$.

In the current study, HP caused severe enamel surface microhardness (SMH) loss and microstructure destruction, which was in agreement with previous studies ${ }^{19,20,22}$. Morphological alternations of the enamel may be predominantly related to the acidity of HP. A combination of the effects of demineralization and reduced organic content of the enamel by HP results in decreased enamel microhardness ${ }^{2}$. By contrast, applying BG before and after bleaching caused milder SMH loss and surface changes, indicating an evident protective effect of BG against HP and it was according to previous study were concluded that $45 S 5$ bioglass applied on bleached enamel led to increase enamel microhardness same as intact enamel23. The modified Bioglass 4555 used in this study is an extremely biocompatible mineral compound that reacts in the aqueous environment and releases ions such as $\mathrm{ca}$ and $\mathrm{Na}$ and $\mathrm{P}$ at great amounts $1,3,8$. When Bioglass particles meet saliva or water, they release sodium, calcium, and phosphorus ions quickly, which stimulates the tooth surface remineralization ${ }^{1}$. A recent study showed that using BG after bleaching could remineralize the enamel surface by increasing the surface $\mathrm{Ca}$ and $\mathrm{P}$ content. Applying BG before bleaching can decrease mineral loss. Precipitation of free calcium and phosphate from BG particles forms a protective calcium phosphate-rich layer on the enamel surface ${ }^{1}$, which is confirmed by the milder porous surface before and after the use of BG with bleaching agents ${ }^{1}$. Saliva induces maximum apatite growth on $B G^{1,6}$ and $B G$ mixed with saliva would produce better protection when applied before and after $\mathrm{HP}^{1}$.

The results of the current study also showed that groups containing BG were able to remineralize the affected enamel surfaces as demonstrated by SEM images. Bioactive glass deposits were found on the enamel surface in BG groups, suggesting that they may act as a source of ions available for remineralization/demineralization areas. This is confirmed by the presence of a protective layer on the enamel surface. The deposits were not removed by thorough washing ${ }^{1}$.

The results of the EDX analysis confirmed mineral loss on the enamel surface of teeth treated with $35 \% \mathrm{HP}$, indicating that bleaching agents caused removal of the organic phase and some of the mineral phase ${ }^{6,24}$. However, the mineral ionic release of BG was much greater in HP compared to DDW, but the difference was not statistically significant, that it can be related to media that samples were stored during treatment.

Within the limitations of this study, like the novelty of topic and lack of similar studies, the results showed that 35\% HP solution caused significant microhardness loss and morphological changes in the enamel. The combination of BG and HP did not alter the whitening efficacy. Moreover, applying BG before and after bleaching prevented enamel microhardness loss optimally and maintained the enamel surface integrity. Additionally, the mineral ionic release of BG was much greater in HP compared to DDW, which could increase the remineralization effect of BG on the bleached enamel. 


\section{References}

1. Deng M, Wen HL, Dong XL, Li F, Xu X, Li H, et al. Effects of $45 S 5$ bioglass on surface properties of dental enamel subjected to 35\% hydrogen peroxide. Int J Oral Sci. 2013 Jun;5(2):103-10. doi: 10.1038/ijos.2013.31.

2. Jiang $T$, Ma $X$, Wang $Y$, Tong $H$, Shen $X$, Hu $Y$, et al. Investigation of the effects of $30 \%$ hydrogen peroxide on human tooth enamel by Raman scattering and laser-induced fluorescence. J Biomed Opt. 2008 Jan-Feb;13(1):014019. doi: 10.1117/1.2870114.

3. Gjorgievska E, Nicholson JW. Prevention of enamel demineralization after tooth bleaching by bioactive glass incorporated into toothpaste. Aust Dent J. 2011 Jun;56(2):193-200. doi: 10.1111/j.1834-7819.2011.01323.x.

4. Cobankara FK, Unlu N, Altinoz HC, Fusun O. Effect of home bleaching agents on the roughness and surface morphology of human enamel and dentine. Int Dent J. 2004 Aug;54(4):211-8.

5. Taha AA, Patel MP, Hill RG, Fleming PS. The effect of bioactive glasses on enamel remineralization: A systematic review. J Dent. 2017 Dec;67:9-17. doi: 10.1016/j.jdent.2017.09.007.

6. Park YH, Choi AN, Jung KH, Park JK, Kwon YH, Son SA. Effects of bioactive glass on microhardness of bleached enamel surface. Korean J Dent Mater. 2018;45(1):1-10. doi: $10.14815 / \mathrm{kjdm}$.2018.45.1.1.

7. Attin T, Vollmer D, Wiegand A, Attin R, Betke H. Subsurface microhardness of enamel and dentin after different external bleaching procedures. Am J Dent. 2005 Feb;18(1):8-12.

8. Rehder Neto FC, Maeda FA, Turssi CP, Serra MC. Potential agents to control enamel caries-like lesions. J Dent. 2009 Oct;37(10):786-90. doi: 10.1016/j.jdent.2009.06.008.

9. Burwell AK, Litkowski LJ, Greenspan DC. Calcium sodium phosphosilicate (NovaMin): remineralization potential. Adv Dent Res. 2009;21(1):35-9. doi: 10.1177/0895937409335621.

10. Andersson $\mathrm{OH}, \mathrm{Kangasniemi} \mathrm{I.} \mathrm{Calcium} \mathrm{phosphate} \mathrm{formation} \mathrm{at} \mathrm{the} \mathrm{surface} \mathrm{of} \mathrm{bioactive} \mathrm{glass} \mathrm{in}$ vitro. J Biomed Mater Res. 1991 Aug;25(8):1019-30.

11. Fredholm YC, Karpukhina N, Brauer DS, Jones JR, Law RV, Hill RG. Influence of strontium for calcium substitution in bioactive glasses on degradation, ion release and apatite formation. J R Soc Interface. 2012 May 7;9(70):880-9. doi: 10.1098/rsif.2011.0387.

12. Fredholm YC, Karpukhina N, Brauer DS, Jones JR, Law RV, Hill RG. Influence of strontium for calcium substitution in bioactive glasses on degradation, ion release and apatite formation. J R Soc Interface. 2012 May 7;9(70):880-9. doi: 10.1098/rsif.2011.0387.

13. Tai BJ, Bian Z, Jiang H, Greenspan DC, Zhong J, Clark AE, et al. Anti-gingivitis effect of a dentifrice containing bioactive glass (NovaMin) particulate. J Clin Periodontol. 2006 Feb;33(2):86-91.

14. Du Min Q, Bian Z, Jiang H, Greenspan DC, Burwell AK, Zhong J, et al. Clinical evaluation of a dentifrice containing calcium sodium phosphosilicate (novamin) for the treatment of dentin hypersensitivity. Am J Dent. 2008 Aug;21(4):210-4.

15. Athanassouli TM, Papastathopoulos DS, Apostolopoulos AX. Dental caries and strontium concentration in drinking water and surface enamel. J Dent Res. 1983 Sep;62(9):989-91.

16. Thuy TT, Nakagaki H, Kato K, Hung PA, Inukai J, Tsuboi S, et al. Effect of strontium in combination with fluoride on enamel remineralization in vitro. Arch Oral Biol. 2008 Nov;53(11):1017-22. doi: 10.1016/j.archoralbio.2008.06.005.

17. Joiner A. The bleaching of teeth: a review of the literature. J Dent. 2006 Aug;34(7):412-9.

18. Paul S, Peter A, Pietrobon N, Hammerle CH. Visual and spectrophotometric shade analysis of human teeth. J Dent Res. 2002 Aug;81(8):578-82. 
19. Jiang $T$, Ma X, Wang Z, Tong $H, H u$ J, Wang Y. Beneficial effects of hydroxyapatite on enamel subjected to $30 \%$ hydrogen peroxide. J Dent. 2008 Nov;36(11):907-14. doi: 10.1016/j.jdent.2008.07.005

20. Josey AL, Meyers IA, Romaniuk K, Symons AL. The effect of a vital bleaching technique on enamel surface morphology and the bonding of composite resin to enamel. J Oral Rehabil. 1996 Apr;23(4):244-50.

21. Soldani P, Amaral CM, Rodrigues JA. Microhardness evaluation of in situ vital bleaching and thickening agents on human dental enamel. Int J Periodontics Restorative Dent. 2010 Apr;30(2):203-11.

22. Xu B, Li Q, Wang Y. Effects of $\mathrm{pH}$ values of hydrogen peroxide bleaching agents on enamel surface properties. Oper Dent. 2011 Sep-Oct;36(5):554-62. doi: 10.2341/11-045-1.

23. Curtis AR, West NX, Su B. Synthesis of nanobioglass and formation of apatite rods to occlude exposed dentine tubules and eliminate hypersensitivity. Acta Biomater. 2010 Sep;6(9):3740-6. doi: 10.1016/j.actbio.2010.02.045.

24. Lee $\mathrm{KH}$, Kim HI, Kim KH, Kwon YH. Mineral loss from bovine enamel by a $30 \%$ hydrogen peroxide solution. J Oral Rehabil. 2006 Mar;33(3):229-33. Dou: 10.1111/j.1365-2842.2004.01360.x 